INTERNACIONAL

\title{
El derecho a la privacidad corporal de los reclusos
}

\author{
The right to body privacy of prisoners
}

\author{
Martina Barroeta \\ Universidad de Chile
}

Yerson Olivares

Universidad de Chile

RESUMEN El derecho a la privacidad es un derecho humano reconocido en diferentes tratados internacionales. Sin embargo, la definición de su contenido y límites -morales y legales- resulta problemática, lo que dificulta su protección. Esta falta de determinación afecta particularmente a grupos humanos que usualmente disfrutan de este derecho de manera reducida, como es el caso de las personas recluidas en establecimientos penitenciarios, lo que muchas veces ha dado pie para vulneraciones de este y otros derechos humanos en su interior. La exposición y en particular la desnudez forzada con el fin de realizar registros corporales o como método de coacción para lograr otros fines, son prácticas comunes en las cárceles del mundo y una forma extendida de vulneración del derecho a la privacidad en ellas. Es por esto que resulta relevante determinar bajo qué supuestos estas prácticas podrían constituir tortura, trato o pena cruel, inhumana o degradante.

PALABRAS CLAVE Privacidad, desnudez forzada, exposición, reclusos, tortura, tratos crueles.

ABSTRACT The Right to Privacy is a human right recognized as such in different international treaties. However, the definition of its content and limits -both legals and morals - is problematic, which hinders its protection. This lack of determination particularly affects some human groups that usually enjoy of this right in a reduced manner, as is the case with people convicted in penitentiary facilities, which has often led to violations of this and other human rights within them. Exposure and, in particular, forced nudity in order to carry out body searches or as a method of coercion to achieve other purposes are common practices in prisons around the world and an extended form of violation of the right to privacy in prisons. This is why it is relevant to determine under 
what assumptions these practices could constitute torture or cruel, inhuman or degrading treatment or punishment.

KEYWORDS Privacy, forced nudity, exposure, prisoners, torture, cruel treatment.

\section{Introducción}

El derecho a la privacidad es un derecho humano consagrado tanto en tratados internacionales como en ordenamientos jurídicos nacionales a nivel constitucional. $\mathrm{Su}$ desarrollo doctrinario y su invocación judicial son profusos y coincidentes en una idea básica de lo que se protege: ciertos ámbitos de la vida de las personas que deben estar libres de la intromisión de terceros. Este resguardo entendido como el «derecho a la privacidad» ha evolucionado hacia una concepción más integral que busca, entre otras cosas, dotar al sujeto de mayor determinación sobre las materias en las cuales este derecho aplica (Solove, 2008).

Dentro de los aspectos que las personas más se afanan por mantener dentro de su esfera de privacidad se encuentra el cuerpo y en especial la desnudez. A pesar de esto, no es común encontrar normas jurídicas que protejan de forma especial la privacidad de la corporalidad, teniendo esta que ampararse la mayoría de las veces en las normas jurídicas generales sobre el derecho a la privacidad.

Esta omisión se torna más preocupante si consideramos que existe un grupo de personas dentro de la sociedad que es particularmente vulnerable a sufrir intromisiones en su cuerpo debido a las circunstancias particulares en las que están obligadas a vivir. Nos referimos a las personas que cumplen condenas privativas de libertad dentro de recintos penitenciarios.

A pesar de que las vulneraciones de este tipo que se comenten en recintos carcelarios son de saber público y han sido ampliamente documentadas, no se han estudiado en profundidad sus características distintivas. La vulneración del derecho a la privacidad del cuerpo del recluso reviste la forma de acto de exposición (Solove, 2008), el cual se puede materializar por distintos medios y con distintos fines. En particular, en este trabajo nos referiremos a las medidas coactivas que implican desnudez y registros corporales.

Ante esta situación, la principal interrogante que se suscita se refiere al nivel de intrusividad de dichas medidas y en qué condiciones se pueden realizar para evitar arbitrariedades. La respuesta a estas preguntas permitiría distinguir, por ejemplo, en qué casos cómo un acto de esta naturaleza es permisible o incluso necesario, y en qué otros es tan pernicioso para el recluso que puede constituir tortura en los términos del artículo 150 A de nuestro Código Penal y del artículo 1 de la Convención contra la Tortura y Otros Tratos o Penas Crueles, Inhumanas o Degradantes. 
De este modo, los objetivos de este trabajo son analizar las particularidades del derecho a la privacidad del cuerpo, caracterizar la desnudez forzada como forma de violación al derecho a la privacidad corporal, exponer críticamente las normas de derecho internacional de los derechos humanos que consagran el derecho a la privacidad y su relación con el cuerpo, analizar el registro corporal y las medidas coactivas que implican desnudez como manifestaciones específicas de violación al derecho a la privacidad del cuerpo que sufren los reclusos y, por último, establecer en qué casos dichas prácticas constituyen tortura o tratos crueles, inhumanos o degradantes.

\section{Privacidad, exposición y cuerpo}

Para efectos de este trabajo, entenderemos la privacidad como cierto ámbito de la vida de las personas que se encuentra reservado del conocimiento de terceros ajenos a ella. ${ }^{1}$ Dicho ámbito puede incluir un cúmulo disímil de actividades, ideas, hechos, objetos, relaciones, etcétera.

De esta forma, los elementos de este concepto son tres. En primer lugar encontramos la parcialidad, es decir, el que solo cierto contenido de la vida de una persona esté sometido a reserva del conocimiento de terceros y no la totalidad. Este elemento, referido a la extensión de la privacidad, recoge la discusión largamente sostenida por la literatura sobre las diferencias conceptuales que existirían entre la intimidad y la privacidad misma (Figueroa, 2014). La primera consistiría en un ámbito reducido dentro de la totalidad de la vida de la persona, que estaría sujeto a un nivel de reserva que ningún agente podría obligar legítimamente a revelar su contenido o acceder a él. Por otro lado, la privacidad sería un ámbito más amplio de la vida de la persona al cual se podría acceder de manera restringida o se podría obligar a la persona a revelar en escenarios determinados por la ley (Figueroa, 2014). Sin embargo, la distinción entre estos dos conceptos es ilusoria toda vez que en la práctica existen normas jurídicas que obligan a revelar o permiten acceder a ambos ámbitos. Por ejemplo, elementos de la vida personal indiscutiblemente considerados íntimos como las fichas médicas, la vida sexual, la vida conyugal o el cuerpo pueden ser objetos de intromisión por parte de agentes del Estado - registros corporales practicados por las policías- o privados - cuando un contrato laboral estipula que el trabajador debe revelar su estado de salud al empleador a través de una ficha médica o diagnóstico que acredite la idoneidad para desempeñar su trabajo- (Lara, Pincheira y Vera, 2014). En conclusión, ambas nomenclaturas, intimidad y privacidad, serían sinónimas (Figueroa, 2014).

En segundo lugar, nos enfrentamos al elemento de reserva. Este elemento es el más esencial del concepto de privacidad. Consiste en el no conocimiento y no difusión de información por persona alguna o por terceros ajenos a ella, entendiendo que los terceros que forman parte de la esfera privada de relaciones de la persona sí podrían tener acceso a los aspectos privados de su vida (Figueroa, 2014). Este elemento 
contempla, en general, tres acciones: que el contenido de la vida personal que corresponde a la privacidad se produzca en el secretismo, que este contenido sea mantenido en esa esfera y que el mismo sea vuelto a ocultar si es revelado.

Por último, debemos tratar el elemento de la multidimensionalidad, el cual se refiere al contenido de la privacidad. Esta es una esfera de la vida de las personas que puede estar compuesta por actividades, ideas, hechos, objetos, relaciones y, en fin, cualquier ámbito que se desee mantener reservado. En este elemento reside una de las razones por las cuales el concepto de privacidad y el derecho que la protege han evolucionado desde el right to be let alone - la no intromisión genérica- a la consideración específica de la vida privada, las comunicaciones, los datos personales, etcétera, como componentes disímiles de la esfera privada de una persona que requieren protección. Este desarrollo se ha asociado al tránsito desde una visión «negativa» - de «libertad frente a la intromisión del Estado»- a una de autodeterminación del sujeto o, dicho de otra forma, la capacidad del sujeto de controlar qué información personal es mantenida y tratada por entes privados y/o públicos. ${ }^{2}$

Daniel J. Solove propone una taxonomía útil que clasifica estas dimensiones y tipifica las formas en que la privacidad puede ser afectada. El autor, considerando que la privacidad es un término «madre» que agrupa una amplia variedad de «cosas», realiza una clasificación de 16 formas de vulneración al derecho a la privacidad que categoriza en cuatro grupos: recolección, procesamiento, diseminación e invasión (Solove, 2006).

En este trabajo nos haremos cargo de la difusión o diseminación de información (Information Dissemination), específicamente de la exposición (Exposure), la que se entiende como el «revelar o exponer la desnudez, el dolor de una persona o funciones corporales» (Figueroa, 2014: 164). La no exposición de estos atributos es considerada por las personas como algo primordial, de modo que su publicación causa humillación y denigración al sujeto expuesto (Solove, 2006).

Para que esta forma de vulneración a la privacidad se configure no es necesario que los atributos expuestos sean únicos del sujeto. Basta con que ellos, a pesar de ser comunes, sean considerados como privados. Ejemplo de esto son los genitales, los actos sexuales o las necesidades orgánicas del cuerpo. La no exposición «protege la dignidad humana como la define la sociedad moderna. La dignidad es parte de ser civilizado, implica la capacidad de trascender la naturaleza animal de uno» (Solove, 2006: 534). En detalle, el disgusto por la exposición se debe a que existen normas predominantemente sociales, de dignidad y decoro, ante las cuales la exposición es una contravención que tiene como sanción la exclusión de la persona de la participación plena en la sociedad.

2. Sin perjuicio de este desarrollo, autores como Solove (2008) consideran que esta concepción resulta limitada en la medida que se refiere principalmente a la información, dejando otros aspectos de la privacidad al descuido. 
De este modo, el cuerpo se consagra como espacio con derecho a la privacidad propio que, por tanto, tiene límites establecidos y que no deberían ser violados.

El cuerpo es el primer ámbito de protección, la cual se da en dos sentidos: el cuerpo como objeto de captación y revelación, y el cuerpo como objeto de invasión física indebida (Figueroa, 2014). De esta forma, la privacidad corporal puede verse vulnerada en casi todos los espacios sociales, lo que refuerza la necesidad de mantener normativa y protocolos estrictos que aseguren la no vulneración de este derecho, sobre todo en espacios donde existen restricciones de libertades como lo son los recintos penitenciarios.

\section{El derecho a la privacidad y derecho a la privacidad del cuerpo: análisis crítico de su reconocimiento internacional}

El reconocimiento general del derecho a la privacidad de la persona en el sistema actual de derechos humanos encuentra su primera expresión en la Declaración Universal de Derechos Humanos de 1948, cuyo artículo 12 declara que nadie será objeto de injerencias arbitrarias en su vida privada, su familia, su domicilio o su correspondencia, ni de ataques a su honra o reputación. Toda persona tiene derecho a la protección de la ley contra estas injerencias o ataques.

Luego, el Pacto Internacional de Derechos Civiles y Políticos de 1966, que establece normas de conducta vinculantes para los Estados parte, dispuso en su artículo 17 que «nadie será objeto de injerencias arbitrarias o ilegales en su vida privada, su familia, su domicilio o su correspondencia, ni de ataques ilegales a su honra y reputación».

Finalmente, el derecho a la privacidad de la persona se encuentra reconocido en términos similares en otros tratados internacionales de derechos humanos, como el artículo 11 de la Convención Americana de Derechos Humanos, el artículo 8 de la Convención Europea de Derechos Humanos y el artículo 16 de la Convención sobre los Derechos del Niño, solo por mencionar algunos.

Analizando en particular la norma contenida en el artículo 17 del Pacto Internacional de Derechos Civiles y Políticos, podemos detectar tres falencias en lo que respecta a nuestro tema. La primera es su énfasis en la protección de lugares - propiedad-, correspondencia —extensible hoy a otros medios de comunicación-y relaciones familiares, mientras que aspectos como el cuerpo son forzados a entenderse dentro de la categoría de «vida privada» que ofrece la norma. Esta fórmula parece insuficiente, considerando que el cuerpo y buena parte de lo relativo a él son ámbitos que generalmente cuentan con un gran nivel de reserva en la vida de las personas, en especial si de su desnudez se trata (Robson, 2013).

La segunda se refiere a los requisitos de ilegalidad o arbitrariedad que fija la norma para que se configure la injerencia vulneratoria del derecho. La doctrina interpretativa que ha establecido el Comité de Derechos Humanos en su Observación 
General 16 sobre el Derecho a la Intimidad establecido en el citado artículo 17 del Pacto Internacional de Derechos Civiles y Políticos señala en su párrafo 4 que:

A juicio del Comité, la expresión «injerencias arbitrarias» puede hacerse extensiva también a las injerencias previstas en la ley. Con la introducción del concepto de arbitrariedad se pretende garantizar que incluso cualquier injerencia prevista en la ley esté en consonancia con las disposiciones, los propósitos y los objetivos del Pacto y sea, en todo caso, razonable en las circunstancias particulares del caso.

Traducida a términos prácticos, la Observación General citada interpreta que cualquier injerencia ilegal a la privacidad de las personas será condenable. Si la injerencia es legal, ella será condenable solo si resulta arbitraria. Esto suscita los problemas usuales al momento de aplicar la norma, entendiendo que tanto la arbitrariedad como su contraparte, la razonabilidad, son criterios de alta carga subjetiva y significativa dependencia casuística.

Por último, llama la atención que cuando la norma que tratamos se refiere a los ataques a la honra y reputación de la persona, solo establezca la ilegalidad como requisito para considerarlos vulneratorios. Esto deja fuera de la esfera de protección del derecho a quienes sufran dichos ataques que, estando amparados en la legalidad, sean arbitrarios.

\section{El derecho a la privacidad del cuerpo del recluso: la desnudez forzada}

El principio de trato humano es el más relevante en lo que respecta al goce y ejercicio de los derechos humanos de las personas privadas de libertad. De él emana la obligación de los Estados de tratar a los reclusos de acuerdo con su dignidad inherente, sin despojarlos de sus derechos fundamentales, exceptuando aquellos de los cuales ha sido privado mediante sentencia condenatoria o los que se ven restringidos como consecuencia necesaria de la privación de libertad. El derecho a la privacidad cabe dentro de esta última categoría (Aguiló, Milos y Nash, 2013).

Sin embargo, debido a este principio, las limitaciones restrictivas de este derecho deben ser estrictas y cumplir con los requisitos de legitimidad que establece el derecho internacional: que la restricción esté contenida en una norma de rango legal, que el objetivo que persigue sea legítimo y que la medida restrictiva adoptada sea proporcional (Aguiló, Milos y Nash, 2013).

En vista de lo que se ha expuesto hasta ahora sobre los problemas que presenta la formulación general del derecho a la privacidad en el Pacto Internacional de Derechos Civiles y Políticos, en especial en lo relativo al cuerpo y a los requisitos de legalidad y arbitrariedad para que se configure la vulneración, además de lo recién expuesto sobre la restricción del derecho a la privacidad de los reclusos, cabe concluir que la situación del derecho a la privacidad de sus cuerpos es un factor de alerta. 
En primer lugar, los establecimientos penitenciarios son espacios donde las condiciones de privacidad de los reclusos se ven reducidas, lo cual puede estimarse como una consecuencia necesaria de su situación dependiendo del caso. En segundo lugar, no es extraño que bajo el mencionado pretexto de la consecuencia necesaria de la privación de libertad tengan lugar las violaciones al derecho a la privacidad del recluso, en especial la del cuerpo. En tercer lugar, la indeterminación de las normas generales sobre el derecho a la privacidad, sumada a la aceptación de que la privacidad del recluso puede afectarse como consecuencia de su situación, deja una zona gris en lo relativo a la privacidad de su cuerpo.

Hemos establecido ya que la exposición es un tipo de vulneración al derecho a la privacidad de la persona que consiste en la revelación de lo relativo a su cuerpo. Luego, hemos descrito a la desnudez forzada como una categoría dentro de la exposición. Por último, en lo que resta de este trabajo abordaremos las formas particulares en que se materializa el despojo forzado de indumentaria que afectan especialmente a los reclusos y que no en todos los casos pueden estimarse como consecuencias necesarias de la privación de libertad.

La desnudez forzada se materializa en su primera forma como medida coactiva dentro de los establecimientos penitenciarios (Rodley, 1995). En este caso, entendemos que la desnudez forzada se perfila como un método para que el recluso preste confesión, sea sancionado por un acto que cometió o se sospecha que ha cometido, para intimidarlo y, en general, para causarle dolor o sufrimiento. Estas dos últimas finalidades son las que más se observan, por ejemplo, en el último informe sobre condiciones de vida dentro de los establecimientos penitenciarios elaborado por el Instituto Nacional de Derechos Humanos, donde los reclusos reportan que el desnudamiento forzado forma parte de las medidas disciplinarias que se les imponen (Instituto Nacional de Derechos Humanos, 2018). Todas estas acciones coinciden con los actos que la definición del artículo 1 de la Convención contra la Tortura consigna como tales.

El registro es la segunda forma en que se puede materializar la exposición y consiste en la exploración del cuerpo del recluso mediante cualquiera de los sentidos, aunque principalmente el tacto, la vista o por medio de otros instrumentos. Si bien el registro corporal generalmente incluye el registro de ropas e incluso puede darse sin despojo de indumentaria, usualmente es un acto que implica la desnudez total o parcial del recluso, en especial cuando se trata de examinar sus cavidades corporales (Robson, 2013). Como resulta evidente, la finalidad del registro corporal de los reclusos es la verificación de que su cuerpo está libre de objetos que puedan poner en riesgo la vida, salud y seguridad de sí mismos y de quienes le rodean, que puedan facilitar su fuga o la comisión de delitos dentro del mismo establecimiento, y que se encuentren prohibidos por las regulaciones de los recintos penitenciarios. 


\section{La desnudez forzada del recluso como tortura y trato o pena cruel, inhumana o degradante}

La prohibición de la tortura y los tratos o penas crueles, inhumanas o degradantes es una norma de ius cogens que se enmarca en el principio de trato humano. Esta prohibición se encuentra principalmente establecida en la Convención Contra la Tortura y Otros Tratos o Penas Crueles, Inhumanas o Degradantes vigente desde 1987. Sus antecedentes directos dentro del sistema internacional de derechos humanos son el artículo 5 de la Declaración Universal de Derechos Humanos, el artículo 7 del Pacto Internacional de Derechos Civiles y Políticos, y la Declaración sobre Protección de Todas las Personas contra la Tortura y Otros Tratos o Penas Crueles, Inhumanas o Degradantes vigente desde 1976.

Existe un vínculo inmediato entre la prohibición de la tortura y los otros tratos o penas crueles, inhumanas o degradantes y el principio de respeto por la dignidad humana, fundamental en el sistema de derechos humanos (Rodley, 2018). De la misma forma, este último se relaciona con el derecho a la privacidad. Esta trinidad de derechos y principios es la base para entender cómo la desnudez forzada puede constituir tortura, trato o pena cruel, inhumana o degradante en el contexto de la reclusión penitenciaria.

Sin embargo, debemos tener en cuenta que nos encontramos ante dos prohibiciones distintas que recaen sobre dos actos distintos. Si bien dichos actos comparten varios aspectos, difieren principalmente en la finalidad con la que deben practicarse para que se constituyan así, a saber, que mientras la tortura se practica para alcanzar los fines tradicionalmente asociados a ella, los tratos o penas crueles, inhumanas o degradantes se practican con otros fines o sin un fin en específico. A pesar de esta distinción, en la que ahondaremos más adelante, sostenemos que las prohibiciones que recaen sobre ambos actos son absolutas, aunque generalmente las normas jurídicas y la literatura solo se enfoquen en la tortura (Rodley, 2018).

Tomando en cuenta estos lineamientos, postulamos que el registro corporal de los reclusos que involucra desnudez total o parcial puede llegar a constituir, bajo ciertas circunstancias, trato cruel, inhumano o degradante, ya que el desnudamiento en este caso se realiza con un fin legítimo, que generalmente tiene que ver con la seguridad del recluso y de la unidad penitenciaria en general, mientras que las medidas coactivas que implican desnudez forzada constituyen siempre tortura, pues se ejecutan con miras a causar dolor o sufrimiento al recluso, ya sea porque esto en sí mismo es la finalidad, ya sea para obtener de él información o castigarlo.

\section{Las medidas coactivas que involucran desnudez como forma de tortura a las personas privadas de libertad}


Anteriormente hemos descrito las medidas coactivas que involucran desnudez en forma coincidente con los actos que consigna el artículo 1, que define la tortura, de La Convención Contra la Tortura y Otros Tratos o Penas Crueles, Inhumanas o Degradantes. Así:

Se entenderá por el término «tortura» todo acto por el cual se inflija intencionadamente a una persona dolores o sufrimientos graves, ya sean físicos o mentales, con el fin de obtener de ella o de un tercero información o una confesión, de castigarla por un acto que haya cometido, o se sospeche que ha cometido, o de intimidar o coaccionar a esa persona o a otras, o por cualquier razón basada en cualquier tipo de discriminación, cuando dichos dolores o sufrimientos sean infligidos por un funcionario público u otra persona en el ejercicio de funciones públicas, a instigación suya, o con su consentimiento o aquiescencia. No se considerarán torturas los dolores o sufrimientos que sean consecuencia únicamente de sanciones legítimas, o que sean inherentes o incidentales a estas.

Se observa que esta definición contempla tres requisitos para que se configure el acto de tortura: que la víctima experimente sufrimiento o dolor mental o físico severo, que dicho sufrimiento o dolor sea causado con la clase de propósito o fin tradicionalmente asociado a la tortura, y que sea, por último, causado en el ámbito de la oficialidad pública (Rodley, 2018).

Si bien los tribunales internacionales de derechos humanos no han fallado de forma unívoca con respecto a lo que diferencia a la tortura del resto de prácticas prohibidas por la Convención citada y el resto de las normas de derecho internacional pertinentes, es de nuestra opinión que el elemento diferenciador clave reside en las finalidades específicas que tiene la tortura, más que en la severidad y naturaleza del dolor o sufrimiento causado a la víctima. Esto llevaría a concebir la tortura como una práctica prohibida independiente de los tratos o penas crueles, inhumanas o degradantes, no como una forma agravada y deliberada de ellas (Rodley, 2018).

Cumpliendo estos requisitos, el acto de despojar mediante la fuerza a un recluso de sus ropas constituirá tortura cuando, además, dicho despojo deje expuestas las zonas del cuerpo que, dependiendo del contexto cultural de la víctima, se consideran censurables a través de la indumentaria, sin importar si la desnudez resultante es total o parcial. En este entendido, el sufrimiento mental o físico que experimenta la víctima de desnudez forzada proviene de una transgresión severa a su privacidad corporal que es imposible de disociar de la connotación sexual de despojar a una persona de sus ropas, más si se toma en cuenta que el encontrarse desnudo frente al victimario puede despertar en la víctima el temor fundado de ser sexualmente agredido. ${ }^{3}$ La desnudez forzada, en consecuencia, no puede calificarse sino como tortura

3. «Istanbul Protocol: Manual on the Effective Investigation and Documentation of Torture and Other 
sexual (Méndez, 2014) que además tiende a traspasar el episodio particular de violencia y tiene repercusiones de esa índole en la vida del recluso, que se manifiestan tanto física como psicológicamente. ${ }^{4}$

Es necesario explicitar, sin embargo, que rara vez la desnudez forzada se practica por sí sola como método de tortura (Rodley, 1995: 167).

\section{El registro corporal practicado al recluso como trato cruel, inhumano o degradante}

El registro corporal es una práctica generalmente regulada en los sistemas jurídicos domésticos que, además, ha sido tratada por diversos organismos de derechos humanos de las Naciones Unidas. En ambos ámbitos, se admite que esta práctica puede ser necesaria para resguardar la seguridad de los reclusos, los gendarmes y del establecimiento penitenciario en general, entre otros fines. Bajo este criterio, el registro corporal puede estimarse como una restricción del derecho a la privacidad que es consecuencia necesaria de la privación de libertad, debido a la relevancia que cobra en este contexto la seguridad y la prevención del crimen.

El problema fundamental cuando hablamos de los registros corporales está en determinar bajo qué circunstancias el registro que se le practica a un recluso constituye un trato cruel, inhumano o degradante.

Definimos los tratos o pena crueles, inhumanas o degradantes como todos esos actos o prácticas realizadas por agentes estatales en el ejercicio de sus funciones, que infligen intencionadamente a una persona dolores o sufrimientos graves, ya sean físicos o mentales, con finalidades distintas a las tradicionalmente asociadas a la tortura o incluso sin ninguna finalidad en particular (Rodley, 2018).

A nuestro juicio, lo que más caracteriza a un registro corporal que implique desnudez como trato cruel, inhumano o degradante, sin embargo, no está presente en la definición. Este es la incompatibilidad del método o forma de registro con la dignidad de la persona recluida a quien se le practica. Este elemento se refiere a la contradicción entre ciertos medios para realizar el registro corporal y el tratamiento que debe darse a los reclusos según el principio del trato humano. Lo anterior se relaciona estrechamente con el requisito de proporcionalidad que deben cumplir las medidas restrictivas de derechos humanos, en el sentido de que debe utilizarse para el registro corporal el medio que menos agrave la dignidad humana y el derecho a la privacidad corporal del recluso.

A este respecto, en el fallo del caso Valašinas con Lituania del 2001, la Corte Europea de Derechos Humanos estimó que mientras los registros corporales que implican

Cruel, Inhuman or Degrading Treatment or Punishment», Oficina del Alto Comisionado de las Naciones Unidas para los Derechos Humanos, 2004, disponible en https://bit.ly/34SDPRW. 
desnudez pueden ser necesarios para asegurar la seguridad del recinto penitenciario o prevenir el desorden o el crimen, ellos deben ser llevados a cabo de una manera apropiada. En detalle, el fallo califica que los hechos del caso - forzar al recluso a desnudarse para ser registrado frente a una persona del sexo opuesto, tocar sus órganos sexuales y comida con las manos sin higienizarlas previamente o aislarlas con guantes- mostraron una clara falta de respeto por la víctima y disminuyeron efectivamente su dignidad humana, dejándola con sentimientos de angustia o inferioridad suficientes para humillarla y degradarla. Bajo este razonamiento, la Corte concluyó que el referido acto había constituido tratamiento degradante en los términos del artículo 3 de la Convención Europea de Derechos Humanos, que prohíbe prácticas de esta naturaleza.

En consonancia con este fallo, la citada Observación General 16 del Comité de Derechos Humanos en su párrafo 8 reafirma la necesidad de tomar medidas que garanticen la dignidad de los registros personales y corporales como lo es el examen por personas del mismo sexo. En la misma línea, la regla 20 de las Reglas de las Naciones Unidas para el tratamiento de las reclusas y medidas no privativas de la libertad para las mujeres delincuentes, sugiere a los Estados que los registros sin ropa y los que sean corporalmente invasivos sean sustituidos por otros métodos de inspección como el escaneo, a fin de evitar sus consecuencias psicológicas dañinas y posibles repercusiones físicas. ${ }^{5}$

\section{Conclusión}

Las afectaciones a la privacidad del cuerpo de los reclusos pueden revestir dos formas principales: la exposición como medida coactiva y la exposición mediante el registro corporal que implique desnudez. En el primer caso, la gravedad de la injerencia puede tener un impacto sobre la persona de la víctima que deberá considerarse como tortura si cumple con los otros requisitos de la definición establecida por el derecho internacional y, además, el acto de despojo deja expuestas zonas de su cuerpo que ordinariamente el recluso mantiene cubiertas por pudor, de acuerdo con su contexto cultural. En el segundo caso, una medida que puede legítimamente ampararse en razones de seguridad de los reclusos puede calificarse como tratos crueles, inhumanos o degradantes si los medios que se utilizan son incompatibles con la dignidad del recluso o la disminuyen.

Este último escenario plantea la necesidad de encontrar un equilibrio entre la seguridad carcelaria y los derechos humanos de los reclusos, propendiendo al uso

5. «Reglas de las Naciones Unidas para el tratamiento de las reclusas y medidas no privativas de la libertad para las mujeres delincuentes (Reglas de Bangkok)», Organización de las Naciones Unidas, 16 de marzo de 2011, disponible en https://bit.ly/35RHoKR. 
de tecnologías que no hagan necesario el desnudamiento total ni parcial de las potenciales víctimas, así como tampoco el contacto físico con su cuerpo, evitando caer en lo que puede configurarse como un trato cruel, inhumano o degradante por parte de agentes estatales. De esta manera, resultaría plausible disminuir la contradicción entre los registros corporales y el tratamiento digno a los cuales los reclusos deben acceder según el principio del trato humano.

En definitiva, este problema visibiliza la urgencia de reconocer al cuerpo y en particular al cuerpo desnudo como objeto prioritario de protección del derecho a la privacidad, lo que se explica por lo inherentemente sensible y sexualmente connotada en términos culturales que resulta la desnudez, acarreando graves consecuencias físicas y sicológicas para el sujeto afectado.

\section{Referencias}

Aguiló, Pablo, Catalina Milos y Claudio Nash (2013). Personas privadas de libertad y medidas disciplinarias en Chile: análisis y propuestas desde una perspectiva de derechos humanos. Santiago: Centro de Derechos Humanos, Universidad de Chile.

Figueroa, Rodolfo (2014). Privacidad. 1. ${ }^{\mathrm{a}}$ ed. Santiago: Universidad Diego Portales.

Instituto Nacional de Derechos Humanos. (2018). Estudio de las condiciones carcelarias en Chile. Santiago: Instituto Nacional de Derechos Humanos.

Lara, Juan Carlos, Carolina Pincheira y Francisco Vera (2014). La privacidad en el sistema legal chileno. Santiago: Derechos Digitales.

MÉNDEZ, Juan E. (2014). Informe del relator especial sobre la tortura y otros tratos o penas crueles, inhumanas o degradantes. Disponible en https://bit.ly/39a695v.

Nash, Claudio (2009). El sistema interamericano de derechos humanos en acción: aciertos y desafíos. México: Porrúa.

Robson, Ruthann (2013). Dressing Constitutionally: Hierarchy, Sexuality, and Democracy from our Hairstyles to our Shoes. 1. $^{\text {a }}$ ed. Nueva York: Cambridge University Press.

Rodley, Nigel S. (1995). Question of the Human Rights of all persons subjected to any form of detention or imprisonment, in particular: torture and other cruel, inhuman or degrading treatment or punishment. Disponible en https://bit.ly/2PWqunb.

-. (2018). Integrity of the Person. In International Human Rights Law. $3 .{ }^{\mathrm{a}}$ ed. Glasgow: Oxford University Press.

Solove, Daniel J. (2006). "A Taxonomy of Privacy». University of Pennsylvania Law Review, 154 (3): 477-560. Disponible en https://bit.ly/2Znj53q.

-. (2008). Understanding Privacy. 1. ${ }^{\mathrm{a}}$ ed. Cambridge: Harvard University Press. 


\section{Sobre los autores}

Martina Barroeta Zalaquett es estudiante de derecho de la Universidad de Chile. Ha trabajado en distintas iniciativas sobre derechos humanos, específicamente en el derecho de la vestimenta, sobre el que expuso recientemente en el Primer Encuentro Internacional de Prácticas Críticas de Moda realizado en el Museo de Arte Contemporáneo de Santiago organizado por el Colectivo Malvestidas. Además, se ha desempeñado como colaboradora de contenido en el área de su especialidad en el sitio web www.modacl.com. Su correo electrónico es martina.barroeta@derecho.uchile.cl.

Yerson Olivares Bonilla es administrador público y licenciado en Ciencias Políticas y Gubernamentales de la Universidad de Chile. Actualmente, se encuentra cursando el diplomado de Ciberseguridad que imparte la Facultad de Derecho de la misma casa de estudios. Se desempeña como profesional de la Subdirección de Alta Dirección Pública de la Dirección Nacional del Servicio Civil. Su correo electrónico es yerson.o.b@gmail.com. 
